\title{
Optimizing within-host viral fitness: infected cell lifespan and virion production rate
}

\author{
Michael A. Gilchrist ${ }^{1}$, Daniel Coombs ${ }^{2}$, Alan S. Perelson* \\ Theoretical Biology and Biophysics Group, MS-K710, Los Alamos National Laboratory, Los Alamos, NM 87545, USA
}

Received 12 February 2004; accepted 8 April 2004

\begin{abstract}
We explore how an infected cell's virion production rate can affect the relative fitness of a virus within a host. We perform an invasion analysis, based on an age-structured model of viral dynamics, to derive the within-host relative viral fitness. We find that for chronic infections, in the absence of trade-offs between viral life history stages, natural selection favors viral strains whose virion production rate maximizes viral burst size. We then show how various life history trade-offs such as that between virion production and immune system recognition and clearance of virally infected cells can lead to natural selection favoring production rates lower than the one that maximizes burst size. Our findings suggest that HIV replication rates should vary between cells with different life spans, as has been suggested by recent observation.
\end{abstract}

Published by Elsevier Ltd.

Keywords: HIV; Viral evolution; Parasite evolution; Viral fitness; Within-host adaptation

\section{Introduction}

Viruses that cause chronic infections, such as HIV and hepatitis $\mathrm{C}$ virus, undergo extensive evolutionary changes during the stationary phase of infection (Martell et al., 1992; Shankarappa et al., 1999; Gretch et al., 1996; Pawlotsky et al., 1998). Within-host adaptation should have implications for the progression of the disease within an individual host as well as for the evolution of the virus across multiple generations of hosts. Most theoretical models addressing within-host viral evolution (e.g. Nowak and May, 1991; Schenzle, 1994; Stilianakis et al., 1997; Regoes et al., 1998) find that natural selection leads to ever increasing replication rates. By taking into account the action of the immune system, Almogy et al. (2002) showed a selective

\footnotetext{
*Corresponding author. Tel.: +1-505-667-5061; fax: + 1-505-6653493.

E-mail address: asp@t10.lanl.gov (A.S. Perelson).

${ }^{1}$ Current address: Department of Biology, University of New Mexico, Albuquerque, NM 87141, USA.

${ }^{2}$ Current address: Department of Mathematics, University of British Columbia, Vancouver, Canada, V6T 1 Z2.
}

advantage for slow reproduction in the presence of a strong immune response.

Here, we use an age-structured model of viral infection to identify the appropriate fitness term for within-host viral evolution during the stationary phase of an infection. Our results indicate that one important component of viral fitness is burst size. Burst size is defined as the expected number of virions produced over the lifetime of an infected cell and is, therefore, directly tied to an infected cell's virion production rate and lifespan. We focus on the question of how a virus can maximize its burst size. We consider first the scenario where the mortality rate of an infected cell is independent of viral production, and then generalize to the case where there is a direct dependence. This latter case seems more realistic since as cells produce viral proteins they become targets for cell-mediated immune responses, and hence their lifespan should be shortened. We then ask how our conclusions are affected if the production rate also affects the ability of a virion to infect a cell or avoid being cleared by the host. For example, increases in protein production rates are likely to lead to increases in transcription, translation, and/or protein folding error rates. Such errors would likely affect virion proteins and 
hence a virus' ability to infect cells or to avoid immunemediated clearance via glycosylation or other means.

\subsection{Model of within-host dynamics}

\subsubsection{The standard model}

Most models of viral infection assume that infected cells produce virions at a constant rate. A simple dynamic model that is commonly used (Perelson et al., 1996; Neumann et al., 1998; Perelson and Nelson, 1999; Nowak and May, 2000) is

$\frac{\mathrm{d} T}{\mathrm{~d} t}=\lambda-k V T-\mathrm{d} T$,

$\frac{\mathrm{d} T^{*}}{\mathrm{~d} t}=k V T-\delta T^{*}$,

$\frac{\mathrm{d} V}{\mathrm{~d} t}=p T^{*}-c V$,

where $T$ is the density of uninfected host cells susceptible to infection, $T^{*}$ is the density of productively infected host cells, and $V$ is the density of free virions within a host. Uninfected cells are created at constant rate $\lambda$, die at rate $d$ per cell and are infected with rate constant $k$. Productively infected cells, $T^{*}$, die at a constant rate $\delta$ and produce virus at a constant rate $p$ per cell. Free virions, $V$, are cleared at a constant rate $c$.

Note that loss of virions by infecting a cell is not explicitly included in this model but is assumed to be part of virion clearance. Below, we present a generalization in which this loss by infection is made explicit. Many modifications of this model are also possible. For example, Perelson et al. (1996) allowed for the possibility of virions being produced in a burst of size $N$ as a cell dies by replacing $p$ by $N \delta$, but with $N$ and $\delta$ constant. In another study, Klenerman et al. (1996) considered a model in which infected cells progress through three discrete states with variable production and mortality rates. More recently, Nelson et al. (2004) introduced an age-structured generalization of the standard model which provides a more flexible and realistic framework for modeling HIV. Such modifications create models with greater flexibility that may better represent the underlying biology of an infection.

\subsubsection{An age-structured model}

Instead of considering discrete states, Nelson et al. (2004) and others (Kirschner and Webb, 1996) have introduced age-structured generalizations of the standard model where the infected $\mathrm{T}$ cell population, $T^{*}$, is structured by the age of the cell's infection $a$, i.e. the amount of time that has lapsed since the cell was infected by an HIV virion. This approach gives greater flexibility in modeling virion production and mortality of an infected $\mathrm{T}$ cell because it allows these parameters to change over the lifetime of an infected cell.
In its most general form, the age-structured model is defined by the partial differential equations

$\frac{\mathrm{d} T}{\mathrm{~d} t}=\lambda-k V(t) T(t)-d T(t)$

$\frac{\partial T^{*}}{\partial t}+\frac{\partial T^{*}}{\partial a}=-(\mu(a)+m) T^{*}(a, t)$,

$\frac{\mathrm{d} V}{\mathrm{~d} t}=\int_{0}^{\infty} p(a) T^{*}(a, t) \mathrm{d} a-k V(t) T(t)-c V(t)$

with the renewal condition for Eq. (5) being

$T^{*}(0, t)=k V(t) T(t)$.

Here, $T^{*}(a, t)$ represents the density per unit age of infected T cells with age of infection $a$ at time $t$, and $p(a)$ represents the virion production rate from infected cells with an infection of age $a$. Further, the death rate of infected cells is broken down into two separate terms, a constant background mortality rate $m$ and an infectiondependent mortality rate $\mu(a)$. If $\mu(a)$ is constant, we have an analogue of the standard model with $\delta=\mu+m$, where $\mu$ is the excess mortality rate due to infection.

From Eqs. (1)-(7) the steady-state densities of virions and infected and uninfected $\mathrm{T}$ cells are found to be

$\hat{T}=\frac{c}{k(N-1)}$,
$\hat{T}^{*}(0)=k \hat{V} \hat{T}$,

$\hat{T}^{*}(a)=\hat{T}^{*}(0) \exp \left[-\int_{0}^{a}(\mu(z)+m) \mathrm{d} z\right]$,

$\hat{V}=\frac{1}{k}\left(\frac{\lambda}{\hat{T}}-d\right)=\frac{\lambda(N-1)}{c}-\frac{d}{k}$,

where the burst size, $N$ is defined by

$N=\int_{0}^{\infty} \exp \left[-\int_{0}^{a}(\mu(z)+m) \mathrm{d} z\right] p(a) \mathrm{d} a$.

This equilibrium is locally stable provided the infection is able to persist within a host (Nelson et al., 2004). Persistence requires a positive equilibrium density of virions, i.e. from Eq. (11)

$\frac{k}{c}(N-1)>\frac{d}{\lambda}$.

The right-hand side of Eq. (13) is the reciprocal of the equilibrium density of $\mathrm{T}$ cells in the absence of infection. As we show below, the left-hand side is an appropriate measure of viral fitness within a host.

\subsection{Viral fitness within a host}

A commonly used measure for viral fitness within a host is the basic reproductive ratio, $R_{0} . R_{0}$ is usually defined as the expected number of secondary infections caused by the introduction of a single infected cell into an uninfected host. After a virion is produced, it may 
either infect a cell with rate $k T$, or be cleared at rate $c$. The probability that a virion will infect a cell is therefore equal to $k T /(k T+c)$. If an infected cell produces $N$ virions and the equilibrium density of (uninfected) $\mathrm{T}$ cells in an uninfected host is $T_{0}=\lambda / d$, then it follows that $R_{0}=N /\left(1+c / k T_{0}\right)$. We show in the appendix that $R_{0}$ is equivalent to the expected number of secondary virions produced by a single virion introduced into an uninfected host, and we employ this definition from now on.

During chronic infection the number of target cells, $T$, can decrease and even be limiting. We now define $R(T)$, a generalization of $R_{0}$, to be equal to the number of expected secondary virions produced from a single virion when the uninfected $\mathrm{T}$ cell density is $T$ and where $R_{0}=R\left(T_{0}\right)$. In the appendix we show that, under the assumption of constant uninfected $\mathrm{T}$ cell density, the net reproductive ratio of a virus is

$R(T)=\frac{N}{1+c / k T}$

from which one can easily see that the persistence criteria, inequality (13), is satisfied if $R_{0}>1$.

In an infected host at equilibrium, the density of virions is neither increasing nor decreasing and, consequently, $R(\hat{T})=1$. However, if the density of uninfected T cells is greater than its equilibrium density $\hat{T}, R(T)$ will be greater than one, and the virus will increase in density. In contrast, if the density of uninfected T cells is less than $\hat{T}, R(T)$ will be less than one and the viral density will decrease over time.

Thus $R(T)$ can be used to calculate the absolute fitness of a virus given the density of uninfected $T$ cells. If a novel viral strain, $n$, is created via mutation within an infected host and the resident viral strain, $r$, is at its equilibrium density, then the absolute fitness of the novel strain is equal to $R(T)$ evaluated at $T=\hat{T}_{r}$. As a result, the novel viral strain $n$ can only increase in frequency if its net reproductive ratio at the resident equilibrium $\mathrm{T}$ cell density, is greater than 1 .

In the appendix we show that the reproductive ratio of the novel, potentially invading, strain, $R_{n}\left(\hat{T}_{r}\right)>1$ if and only if $\left(k_{n} / c_{n}\right)\left(N_{n}-1\right)>\left(k_{r} / c_{r}\right)\left(N_{r}-1\right)$, where subscripts $n$ and $r$ denote properties of the novel and resident strains, respectively. This inequality defines the viral invasion criteria within a host and, therefore, implies that natural selection will favor viral strains which maximize the quantity $(k / c)(N-1)$. We therefore define a virus's relative fitness within a host, $w$, as

$w=\frac{k}{c}(N-1)$.

The maximization of (relative) viral fitness, $w$, will depend on the relationship between the terms in Eq. (15). Assuming independence, it follows that natural selection favors viral strains that minimize their clearance rate, maximize their infection rate, and maximize their burst size. However, if these terms are not independent, then maximization of $w$ is more complex.

\subsection{Maximizing fitness via virion production rate}

We now explore how the virion production rate, $p$, can evolve to maximize viral fitness. Burst size, $N$, is a monotonic function of $p$, so here we consider the problem of finding the production rate which maximizes $N$.

\subsubsection{Production-independent mortality}

In the standard model, Eqs. (1)-(3), both the infected cell mortality and the virion production rates are fixed over the lifespan of a cell. Under these assumptions

$N=\int_{0}^{\infty} p \exp [-(m+\mu) a] \mathrm{d} a=\frac{p}{m+\mu}$.

Here, virus strategies that increase $p$ and decrease $\mu$ lead to greater burst sizes $N$. Assuming that there is some physiological maximum rate of virion production, $p_{\max }$, we expect the virus to evolve to produce virions at rate $p_{\max }$ and to reduce its impact on cell mortality, i.e. reduce $\mu$. Further, Eq. (13) shows that the maximum viral production rate, $p_{\max }$, must be greater than $(m+$ $\mu)((d / \lambda)(c / k)+1)$, otherwise the infection will be unable to persist within the host.

\subsubsection{Production-dependent mortality}

There are a number of reasons to expect an infected cell's excess mortality rate, $\mu$, to be a function of the virion production rate, $p$. For example, in the process of replicating, a virus utilizes the resources of its host cell in order to produce viral proteins. The loss of cell resources and possible cytotoxic effects of viral proteins are likely to lead to an increase in the cell's death rate (Schneider and Shenk, 1987; Lenardo et al., 2002; Gustin and Sarnow, 2001). Further, assuming that the virion production rate is positively correlated with the density of viral peptides within the cytosol, one would expect the number of cell surface MHC-I molecules presenting viral peptides to increase with viral production rate, leading to an increase in the infected cell's death rate via cytotoxic $\mathrm{T}$ cell activity. Experimental evidence supports this hypothesis (Vijh et al., 1998).

In this scenario, with $p$ still considered to be a constant, Eq. (12) is revised to

$N=\int_{0}^{\infty} p \exp [-(\mu(p)+m) a] \mathrm{d} a=\frac{p}{\mu(p)+m}$.

This formulation exposes a trade-off between current and future virion production. Assuming $\mu$ increases with $p$, higher current virion production, i.e. higher $p$, reduces the value of future virion production by reducing the 
probability that a cell will remain alive. As a result, manipulating the host cell to produce virions at the maximum rate, $p_{\max }$, may no longer necessarily lead to the maximization of burst size.

Differentiating $N$ with respect to $p$, we find that at the optimal production rate, $p^{*}$,

$\mu^{\prime}\left(p^{*}\right)=\frac{\mu\left(p^{*}\right)+m}{p^{*}}$

and $\mu^{\prime \prime}\left(p^{*}\right)>0$ (Sasaki and Iwasa, 1991; Coombs et al., 2003). Here' denotes differentiation with respect to $p$. A sufficient condition for a solution to Eq. (18) to exist is that $\mu(p)$ is increasing and $\mu^{\prime \prime}(p)>z$ for some constant $z>0$ (for additional details, see Coombs et al., 2003). If $p^{*}$ is bigger than $p_{\max }$, then $p^{*}$ will not be achieved and instead the production rate should evolve to the boundary value $p_{\max }$.

If $\mu(p)$ is concave up (and satisfies $\mu^{\prime \prime}(p)>z>0$ ), $N$ is no longer an strictly increasing function of $p$ (Fig. 1c) and the optimal production rate can be calculated by finding the production rate that satisfies Eq. (18). In cases where Eq. (18) cannot be satisfied (e.g. linear and concave down forms of $\mu(p)), N$ is a strictly increasing function of $p$ and is maximized at $p_{\max }$ (Figs. 1a and b).

Regardless of the form of $\mu(p)$, in order for the viral infection to persist, the maximum burst size must still satisfy inequality (13). Substituting $p /(\mu(p)+m)$ for $N$ in Eq. (13), we find that the additional mortality function due to infection, $\mu(p)$, must be less than $p(1+$ $(c / k)(d / \lambda))^{-1}-m$ at $p=p^{*}$ in order for the virus to persist within the host (see the gray shading in Fig. 1c). The persistence criteria become more restrictive with increases of the clearance rate $c$ and less restrictive with increases of the rate constant $k$.

\subsubsection{Allowing dynamic production schedules}

We now generalize by allowing virion production to vary over the infection period, i.e. consider the case in which $p=p(a)$. A dynamic production schedule allows for more realistic descriptions of the biology underlying the infection of a cell. For example, cells do not begin producing virions immediately upon infection, thus one can assume that $p(0)=0$. It can be shown that if $\mu$ is not an explicit function of infected cell age, but an implicit one, i.e. $\mu(a)=\mu(p(a))$, then the optimal virion production schedule is to produce virions at a constant rate $p^{*}$, where $p^{*}$ is the optimal production rate in the $p=$ constant case (Coombs et al., 2003). As pointed out by Sasaki and Iwasa (1991), given a non-zero optimal production rate, $p^{*}$, and the assumption that $p(0)=0$, natural selection will favor viruses which reach $p^{*}$ the quickest. Thus even if $p(0)$ is constrained to be zero, all of our previous conclusions provide a good approximation to the case in which virion production can vary with time of infection, $a$, provided $\mu$ itself is not an explicit function of $a$.

\subsubsection{The effect of background mortality on $p^{*}$}

Focusing on the case in which burst size $N$ is maximized at some intermediate production rate $p^{*}$ (e.g. Fig. 1c), we can rewrite Eq. (18) to get an implicit equation for $p^{*}$,

$p^{*}=\frac{\mu\left(p^{*}\right)+m}{\mu^{\prime}\left(p^{*}\right)}$.
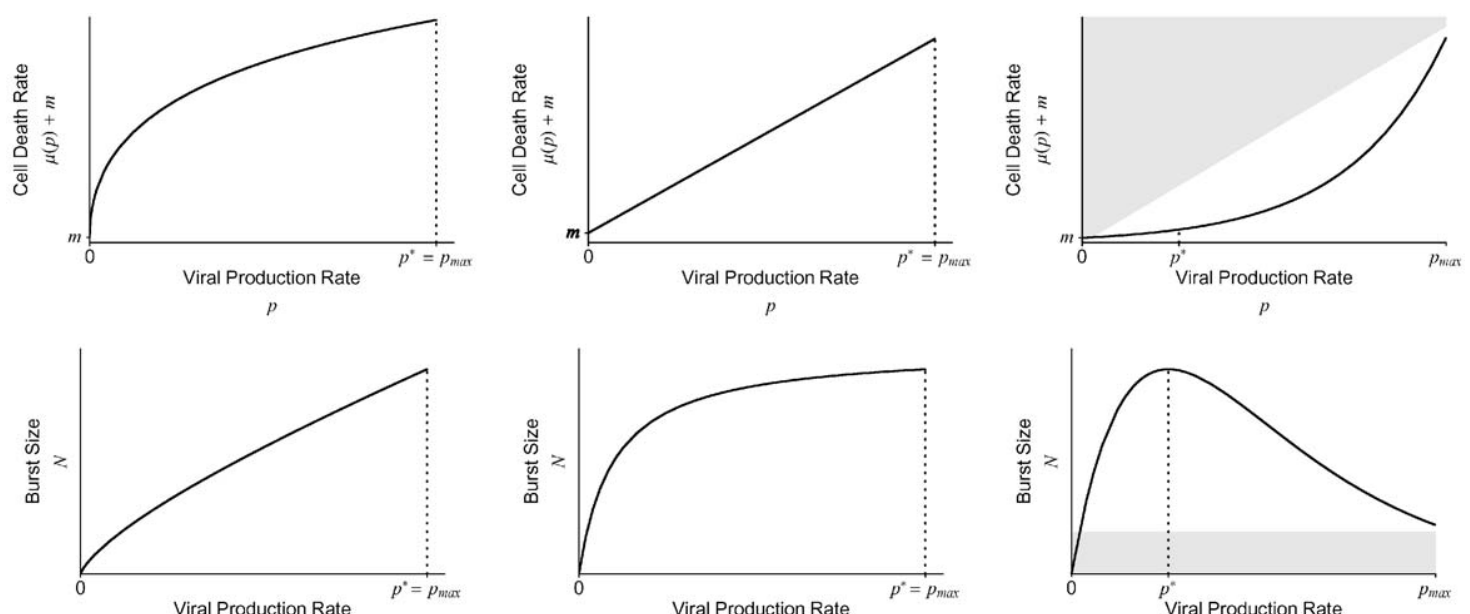

(a)

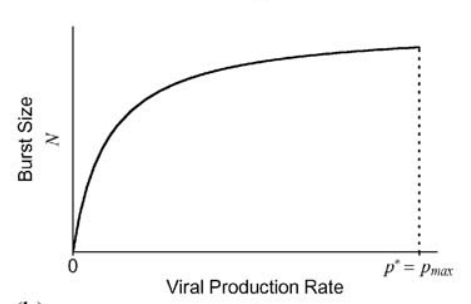

(b)

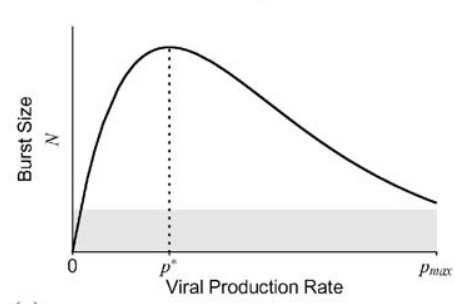

(c)

Fig. 1. Illustration of how infected cell death rate $(\mu(p)+m)$ and burst size $N$ change with viral production rate $p$ for three different relationships between $\mu$ and $p$. The optimal production rate $p^{*}$ maximizes burst size, $N$, within the biologically possible range of production rates, 0 to $p_{\max }$. (a), (b), and (c) illustrate how production-dependent cell death rate, $\mu(p)$, and burst size $N$ change with $p$ when $\mu(p)$ is a decelerating, linear, or an accelerating function of $p$, respectively. In the decelerating and linear case, $N$ is an ever increasing function of $p$. Consequently, burst size is maximal at $p_{\max }$. In contrast, in the accelerating case then there is a finite virion production rate at which burst size is maximized. The shaded areas indicate regions in which the persistence criteria in (13) is not met and, therefore, the infection will be unable to persist. 


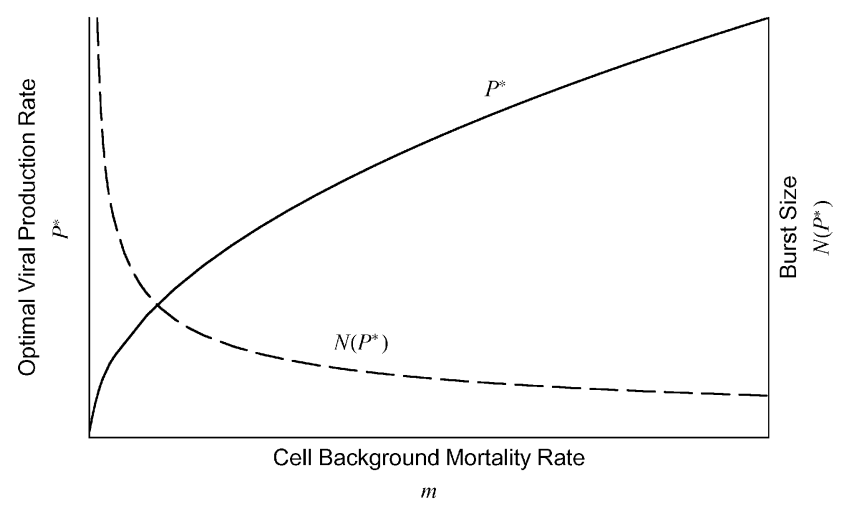

Fig. 2. Effect of the host cell's background mortality rate $m$ on optimal static production rate $p^{*}$.

Differentiating Eq. (19) with respect to the cell's background mortality rate, $m$, yields

$\frac{\mathrm{d} p^{*}}{\mathrm{~d} m}=\frac{1}{\mu^{\prime}\left(p^{*}\right)}=\frac{p^{*}}{\mu\left(p^{*}\right)+m}=N\left(p^{*}\right)$.

Thus, as the background mortality rate $m$ increases, the best virion production rate $p^{*}$ increases, but at a decreasing rate. Further, the optimal burst size $N\left(p^{*}\right)$ decreases asymptotically towards zero as $m$ increases (Fig. 2). This behavior implies that long-lived cells (small $\mathrm{m}$ ) should have greater burst sizes than shortlived cells (large $m$ ) even though the optimal virion production rate in short-lived cells is greater.

\subsubsection{Linking production to other terms}

So far, we have assumed that the virion infection and clearance rates, $k$ and $c$, respectively, are constants unaffected by the virion production rate. However, these rates may vary with $p$. For example, increasing protein translation rates has been shown to increase the probability a protein is misfolded (Komar et al., 1999; Cortazzo et al., 2002). Thus assuming that misfolded proteins are not all destroyed and consequently that some are used by the virus, misfolding can lead to increases in $c$ and reductions in $k$. If a virus has adapted to reduce the antigenicity of its envelope proteins, misfolded proteins could lead to greater antibody binding to free virus, which would in turn lead to an increase in the virion's average clearance rate $c$. Similarly, misfolded envelope proteins would be expected to have a lower target cell binding rate, thus directly decreasing $k$. Finally, misfolded viral proteins involved in post cell-invasion processes, such as reverse transcription, should have lower enzymatic activities and shorter intracellular half-lives (and hence concentrations). Thus, misfolded proteins could also indirectly reduce the average rate of successful infection, $k$. The effect of reducing the intracellular half-life of hepatitis B virus core protein has recently been reported to have a substantial antiviral effect (Deres et al., 2003).
Eq. (15) shows that viral fitness depends on the ratio of $k$ to $c$. If an increase in production rate leads to either an increase in the clearance rate, a decrease in the infection rate or both, the overall effect is a reduction in viral fitness. Writing $z(p)=k(p) / c(p)$, it follows that $w=$ $z(p)(N(p)-1)$ and

$\frac{\mathrm{d} w}{\mathrm{~d} p}=z^{\prime}(N-1)+N^{\prime} z$,

where' denotes differentiation with respect to $p$. Eq. (21) illustrates that maximizing burst size, $N$, only maximizes viral fitness, $w$, if the infection-clearance ratio is invariant as assumed in the previous section, i.e. $z^{\prime}=$ 0 . If the infection-clearance ratio decreases with production rate, $z$ will decrease with increasing production rate, i.e. $z^{\prime}<0$ and thus viral fitness is maximized at a production rate below that which maximizes burst size.

This effect is clearly illustrated when a cell's death rate is independent of its virion production rate, i.e. $\mu(p)=0$. In this case there is no trade-off between virion production and cell mortality so burst size is an increasing function of $p$. Thus if $z$ is independent of $p$, increasing $p$ will always lead to an increase in viral fitness. However, if $z$ is dependent on $p$ then viral fitness may be maximized at an intermediate production rate. For example, if $z(p) \propto p^{-b}$, where $b>1$, then the optimal production rate is $p^{*}=(b m) /(b-1)$. Thus provided that $p_{\max }$ is sufficiently large, the optimal production rate will be less than $p_{\max }$. More generally, the impact of production on the infection-clearance ratio $z$ leads to a optimal production rate less than $p_{\max }$ so long as the inequality $-z^{\prime}(p)(N-1) / z>N^{\prime}(p)$ holds at $p=p_{\max }$.

\section{Discussion}

We used a generalized model of HIV-1 infection to show that natural selection within a host favors viruses that maximize the relative fitness $w=(k / c)(N-1)$. Consequently, in the absence of any interdependence among the parameters in $w$, natural selection should favor viral strains that maximize burst size, $N$, and the rate at which they infect uninfected cells, $k$. Simultaneously, natural selection should favor viral strains that minimize their virion clearance rate from the host, $c$. Thus, for example, to the extent that antibody responses to the virus help clear the virus or neutralize it, one would expect natural selection to favor "escape mutants" that minimize antibody binding, since antibody binding can increase clearance and reduce infectivity.

In our model, the rate of $\mathrm{T}$ cell production, $\lambda$, was assumed to be a constant. However, other models, which explicitly include $\mathrm{T}$ cell proliferation have replaced $\lambda$ by a density dependent function, such as a 
logistic function (Kepler and Perelson, 1998). Including such a proliferation term has no qualitative effect on our overall results. This is, in part, because the definition of viral fitness we derive is independent of how $\mathrm{T}$ cells are regulated. Another plausible scenario is that $\lambda$ changes with $V$ or $T^{*}$ as in models that account for thymic infection by reducing the source of new T cells (Perelson et al., 1993). We leave the formal exploration of this possibility for future studies. We do, however, note that if such a scenario exists and there is an upper bound on $\lambda$ then it seems likely that the system will be driven to this maximum $\mathrm{T}$ cell production rate. If this happens, then our current analysis should be applicable to this situation.

One of the key parameters for maximizing burst size, $N$, is the cell's background mortality rate, $m$. In the case of HIV-1, this rate varies between different types of cells susceptible to infection. Based on our finding that higher production rates are favored in cells with higher mortality rates, we would expect low production rates in cells with low background mortality rates, such as resting $\mathrm{T}$ cells and macrophages, and high production rates in cells with high-background mortality rates, such as activated $\mathrm{T}$ cells. Measurements of intracellular viral RNA levels in resting and activated CD4 $+\mathrm{T}$ cells support this prediction (Zhang et al., 1999). This difference in intracellular viral RNA concentration might be achieved by different strains of HIV that are tropic for different cell types, e.g. macrophage tropic or $\mathrm{R} 5$ viruses and $\mathrm{T}$ cell tropic or $\mathrm{X} 4$ viruses, having different replication rates (Fenyö et al., 1988) or by a single strain if it is able to adjust its replicative rate in different cell types, although one would also expect the availability of intracellular resources to affect viral replication rates.

We note that our model is not particular to HIV. If one considers hepatitis $\mathrm{C}$ virus (HCV) that infects hepatocytes, which are long-lived cells with an estimated half-life of 300 days in the normal liver (MacSween et al., 1987), our theory predicts these cells will produce $\mathrm{HCV}$ at low rates. This is consistent with the low levels of intracellular HCV RNA (mean 20-40 copies) observed in infected hepatocytes (Gosalvez et al., 1998). Further, since hepatocytes are metabolically active cells it is unlikely that all transcription is down regulated in these cells, suggesting that $\mathrm{HCV}$ may be independently regulating its replication to optimize its fitness.

In addition to the cell's background mortality rate, the maximization of viral burst size, $N$, is also a function of the relationship between the virion production rate $p$ and the infection-dependent mortality rate $\mu$. If the infection-dependent mortality rate $\mu$ is a concave up function of the virion production rate $p$, then within host viral fitness is maximized at an intermediate virion production rate, $p^{*}$. Dependence of the virion's infection rate constant, $k$, and/or its clearance rate, $c$, on the virion production rate, $p$, can also affect the optimal virion production rate, $p^{*}$. Trade-offs between the ratio $k / c$ and $p$ may lead to within host viral fitness being maximized at intermediate production rates even in the absence of a direct relationship between virion production and cell mortality. This finding is conceptually similar to the findings of Day (2001) where morbidity effects can restrict disease evolution even in the absence of direct mortality effects.

While it is plausible that $\mu(p)$ is concave up, the exact form of $\mu(p)$ may vary between hosts and even over the lifespan of the host. For example, the efficacy of the immune system in attacking infected cells will vary between hosts. Such variation can lead to variation in the exact form of $\mu(p)$ and consequently variation in the optimal virion production rate between hosts. Further, if the efficacy of the immune system changes during the course of a long lived chronic viral infection, we would expect the optimal virion production rate to change as well.

The arguments presented here also support a mechanism of HIV pathogenesis by which long term adaptation of HIV-1 to an individual host could play a direct role in the eventual breakdown of the host's immune system. While we have not modeled it here, because uninfected $\mathrm{T}$ cells, $\hat{T}$, play a role as helper cells in generating immune responses, it is likely that the production-dependent mortality rate of an infected cell could be affected by the density of $\hat{T}$. If it is the case that $\mu$ is a function of both $p$ and $\hat{T}$ (not just $p$ as we have assumed) a decrease in $\hat{T}$ will lead to a decrease in the efficacy of the host's immune system which, in turn, could lead to an increase in the optimal HIV-1 virion production rate. This is because maximizing the fitness term $w$, given by Eq. (15) is equivalent to minimizing the equilibrium density of uninfected $\mathrm{T}$ cells, $\hat{T}$, given by Eq. (8). Consequently, within host adaptation by HIV-1 should lead to a decrease in $\hat{T}$. At first such a decrease in $\hat{T}$ may not have a measurable effect on the efficacy of the host's immune response to the infection. However, any small decrease in immune response efficacy translates to a correspondingly small increase in the optimal production rate and consequently in burst size, $N$. A slight increase in $N$ leads in turn to another slight decrease in $\hat{T}$. As long as the relationship between immune system efficacy and $\hat{T}$ is essentially flat, i.e. $\partial \mu / \partial \hat{T} \approx 0$, this feedback process could progress quite slowly. However, if $\hat{T}$ decreases into a region in which efficacy changes rapidly with $\hat{T}$, then we would expect this feedback process to lead to a precipitous decline in $\hat{T}$ and consequently the host's ability to fight off other infections. Gaining a better understanding how immune response function varies with $\mathrm{T}$ cell density (i.e. how $\mu$ changes with $\hat{T}$ ) should allow a more formal exploration of this hypothesis in future studies. 
Viruses such as HIV and hepatitis C undergo significant evolutionary change during the course of chronic infection. However, such changes can be neutral, deleterious or adaptive. Having the appropriate measure of viral fitness within a host is critical to understanding if and how a virus may adapt to its host. Not only will within host adaptation have implications for the progression of the disease over the lifetime of the host, but it also is likely to have important implications for the long term adaptation of a virus to its host population.

\section{Acknowledgements}

Portions of this work were performed under the auspices of the US Department of Energy and supported under Contract W-7405-ENG-36, and NIH Grants RR06555 and AI28433. M.A.G. was also partially supported by NIH Grant GM063882-01 to A. Wagner. We thank J. Percus for valuable discussions.

\section{Appendix. Maximizing burst size $N$}

We compute the net reproductive ratio, $R$, as a function of life history parameters, $c, k, m, p(a)$ and $\mu(a)$, by calculating the expected number of secondary virus particles produced by a single virion introduced at time $t=0$. Let $p_{v}(t)$ be the probability that a virion introduced at time $t=0$ survives until time $t$. According to Eq. (6), virion survival is affected by background virion clearance and the "loss" of the particle through the infection of a $\mathrm{T}$ cell. These effects are described by the equation

$\frac{\mathrm{d} p_{v}}{\mathrm{~d} t}=-(c+k T) p_{v}$.

Solving Eq. (A.1) with the initial condition $p_{v}(0)=1$ yields

$p_{v}(t)=\exp \left[-\left(k \int_{0}^{t} T(z) \mathrm{d} z+c t\right)\right]$.

Each infection of a $\mathrm{T}$ cell is expected to produce $N$ additional virions. Thus the number of secondary virions produced, which we call $W(t)$, is given by

$W(t)=\int_{0}^{t} p_{v}(t) k T(t) N \mathrm{~d} t$.

The net reproductive ratio of a virion, $R(T)$, is the number of virions produced in the limit of $t$ approaching infinity, i.e.

$R(T)=t \rightarrow \lim _{\infty} W(t)$.

If we assume that the density of uninfected $\mathrm{T}$ cells is constant over the times involved in infection of a cell and the production of a new generation of new virions, then

$R(T)=k T N \int_{0}^{\infty} p_{v}(t) \mathrm{d} t=\frac{N}{1+c / k T}$.

If the density of $\mathrm{T}$ cells is equal to the equilibrium density $\hat{T}$, Eq. (8), then $R(\hat{T})=1$. At equilibrium each virus particle is expected to lead to the production of exactly one new virus particle.

Invasion criterion: In order for a novel viral strain $n$ to invade a resident strain $r$ its fitness upon introduction into the system must be greater than 1. Thus the invasion criteria for a novel strain is simply

$R_{n}\left(\hat{T}_{r}\right)=\frac{N_{n}}{1+c_{n} / k_{n} T_{r}}>1$,

where the subscripts $n$ and $r$ refer to parameters relevant to the novel and resident strains respectively. Using Eq. (8) to substitute for $\hat{T}_{r}$ it follows that

$\frac{k_{n}}{c_{n}}\left(N_{n}-1\right)>\frac{k_{r}}{c_{r}}\left(N_{r}-1\right)$

or, equivalently, $1 / \hat{T}_{n}>1 / \hat{T}_{r}$. Because the invasion criteria indicates that the viruses that have greater values of the term $(k / c)(N-1)$ will be favored by natural selection, we define the relative fitness of a virus, $w$, as

$w=\frac{k}{c}(N-1)$.

\section{References}

Almogy, G., Cohen, N., Stöcker, S., Stone, L., 2002. Immune response and virus population composition: HIV as a case study. Proc. R. Soc. London B 269, 809-815.

Coombs, D., Gilchrist, M.A., Percus, J., Perelson, A.S., 2003. Optimal viral production. Bull. Math. Biol. 65, 1003-1023.

Cortazzo, P., Cervenansky, C., Marin, M., Reiss, C., Ehrlich, R., Deana, A., 2002. Silent mutations affect in vivo protein folding in Escherichia coli. Biochem. Biophys. Res. Comm. 293, 537-541.

Day, T., 2001. Parasite transmission modes and the evolution of virulence. Evolution 55, 2389-2400.

Deres, K., Schroder, C.H., Paessens, A., Goldmann, S., Hacker, H.J., Weber, O., Kramer, T., Niewohner, U., Pleiss, U., Stoltefuss, J., Graef, E., Koletzki, D., Masantschek, R.N.A., Reimann, A., Jaeger, R., Gross, R., Beckermann, B., Schlemmer, K.H., Haebich, D., Rubsamen-Waigmann, H., 2003. Inhibition of hepatitis B virus replication by drug-induced depletion of nucleocapsids. Science 299, 893-896.

Fenyö, E.M., Morfeldtmanson, L., Chiodi, F., Lind, B., Vongegerfelt, A., Albert, J., Olausson, E., Asjo, B., 1988. Distinct replicative and cytopathic characteristics of human immunodeficiency virus isolates. J. Virol. 62, 4414-4419.

Gosalvez, J., Rodriguez-Inigo, E., Ramiro-Diaz, J.L., Bartolome, J., Tomas, J.E., Oliva, H., Carreno, V., 1998. Relative quantification and mapping of hepatitis $\mathrm{C}$ virus by in situ hybridization and digital image analysis. Hepatology 27, 1428-1434.

Gretch, D.R., Polyak, S.J., Wilson, J.J., Carithers, R.L., Perkins, J.D., Corey, L., 1996. Tracking hepatitis C virus quasispecies major and 
minor variants in symptomatic and asymptomatic liver transplant recipients. J. Virol. 70, 7622-7631.

Gustin, K., Sarnow, P., 2001. Effects of poliovirus infection on nucleocytoplasmic trafficking and nuclear pore complex composition. EMBO J. 20, 240-249.

Kepler, T.B., Perelson, A.S., 1998. Drug concentration heterogeneity facilitates the evolution of drug resistance. Proc. Natl Acad. Sci. 95, 11514-11519.

Kirschner, D., Webb, G.F., 1996. A model for treatment strategy in the chemotherapy of AIDS. Bull. Math. Biol. 58, 367-390.

Klenerman, P., Phillips, R., Rinaldo, C., Wahl, L., Ogg, G., May, R., McMichael, A., Nowak, M., 1996. Cytotoxic T lymphocytes and viral turnover in HIV type 1 infection. Proc. Natl Acad. Sci. 93, $15323-15328$.

Komar, A., Lesnik, T., Reiss, C., 1999. Synonymous codon substitutions affect ribosome traffic and protein folding during in vitro translation. FEBS Lett. 462, 387-391.

Lenardo, M.J., Angleman, S.B., Bounkeua, V., Dimas, J., Duvall, M.G., Graubard, M.B., Hornung, F., Selkirk, M.C., Speirs, C.K., Trageser, C., Orenstein, J.O., Bolton, D.L., 2002. Cytopathic killing of peripheral blood CD4(+) T lymphocytes by human immunodefficiency virus type 1 appears necrotic rather than apoptotic and does not require env. J. Virol. 76, 5082-5093.

MacSween, R.N.M., Anthony, P.P., Scheuer, P.J., Burt, A.D., Portmann, B., 1987. In: MacSween, R.N.M., Anthony, P.P., Scheuer, P.J. (Eds.), Pathology of the Liver. Churchill Livingstone, New York

Martell, M., Esteban, J.I., Quer, J., Genesca, J., Weiner, A., Esteban, R., Guardia, J., Gomez, J., 1992. Hepatitis-C virus (HCV) circulates as a population of different but closely related genomes-quasi-species nature of $\mathrm{HCV}$ genome distribution. J. Virol. 66, 3225-3229.

Nelson, P.W., Gilchrist, M.A., Coombs, D., Hyman, J.M., Perelson, A.S., 2004. An age-structured model of HIV infection that allows for variations in the production rate of viral particles and the death rate of productively infected cells, Math. Biosci. Eng., in press.

Neumann, A.U., Lam, N.P., Dahari, H., Gretch, D.R., Wiley, T.E., Layden, T.J., Perelson, A.S., 1998. Hepatitis C viral dynamics in vivo and the antiviral efficacy of interferon-alpha therapy. Science 282, 103-107.

Nowak, M.A., May, R.M., 1991. Mathematical biology of HIV infections-antigenic variation and diversity threshold. Math. Biosci. 106, 1-21.
Nowak, M.A., May, R.M., 2000. Virus Dynamics: Mathematical Principles of Immunology and Virology. Oxford University Press, New York.

Pawlotsky, J.M., Pellerin, M., Bouvier, M., Roudot-Thoraval, F., Germanidis, G., Bastie, A., Darthuy, F., Remire, J., Soussy, C.J., Dhumeaux, D., 1998. Genetic complexity of the hypervariable region 1 (HVR1) of hepatitis C virus (HCV): influence on the characteristics of the infection and responses to interferon alfa therapy in patients with chronic hepatitis C. J. Med. Virol. 54, 256-264.

Perelson, A.S., Neumann, A., Markowitz, M., Leonard, J., Ho, D., 1996. HIV-1 dynamics in vivo: virion clearance rate, infected cell life-span, and viral generation time. Science 271, 1582-1586.

Perelson, A.S., Nelson, P.W., 1999. Mathematical analysis of HIV-1 dynamics in vivo. SIAM Rev. 41, 3-44.

Perelson, A.S., Kirschner, D.E., Boer, R.J., 1993. Dynamics of HIV infection of $\mathrm{CD}^{+} \mathrm{t}$ cells. Math. Biosci. 114, 81-125.

Regoes, R.R., Wodarz, D., Nowak, M.A., 1998. Virus dynamics: the effect of target cell limitation and immune responses on virus evolution. J. Theor. Biol. 191, 451-462.

Sasaki, A., Iwasa, Y., 1991. Optimal growth schedule of pathogens within a host: switching between lytic and latent cycles. Theor. Popul. Biol. 39, 201-239.

Schenzle, D., 1994. A model for AIDS pathogenesis. Stat. Med. 13, 2067-2079.

Schneider, R., Shenk, T., 1987. Impact of virus-infection on host-cell protein-synthesis. Annu. Rev. Biochem. 56, 317-332.

Shankarappa, R., Margolick, J.B., Gange, S.J., Rodrigo, A.G., Upchurch, D., Farzadegan, H., Gupta, P., Rinaldo, C.R., Learn, G.H., He, X., Huang, X.L., Mullins, J.I., 1999. Consistent viral evolutionary changes associated with the progression of human immunodefficiency virus type 1 infection. J. Virol. 73, 10489-10502.

Stilianakis, N.I., Dietz, K., Schenzle, D., 1997. Analysis of a model for the pathogenesis of AIDS. Math. Biosci. 145, 27-46.

Vijh, S., Pilip, I.M., Pamer, E.G., 1998. Effect of antigen-processing efficiency on in vivo $\mathrm{T}$ cell response magnitudes. J. Immunol. 160, 3971-3977.

Zhang, Z., Schuler, T., Zupancic, M., Wietgrefe, S., Staskus, K., Reimann, K., Reinhart, T., Rogan, M., Cavert, W., Miller, C., Veazey, R., Notermans, D., Little, S., Danner, S., Richman, D., Havlir, D., Wong, J., Jordan, H., Schacker, T., Racz, P., TennerRacz, K., Letvin, N., Wolinsky, S., Haase, A., 1999. Sexual transmission and propagation of SIV and HIV in resting and activated CD4(+) T cells. Science 286, 1353-1357. 